\title{
Dynamic Spectrum Management System Aggregating Non-Contiguous Spectrum over TVWS
}

\author{
Yuying Dai, Angelo Cuffaro, Jean-Louis Gauvreau, Rocco Di Girolamo, Athmane Touag, Alpaslan \\ Demir \\ InterDigital Communications, LLC \\ 1000 Sherbrooke Street West, Montréal, Québec, Canada, H3A 3G4
}

\begin{abstract}
This paper proposes a wireless communication system comprising a DSM (Dynamic Spectrum Management) Engine and DSM clients that can dynamically and opportunistically use and aggregate TVWS spectrum when deployed in a local area such as a home or a small office. Operation in TVWS spectrum requires technology that can address the sparse and non-contiguous nature of TVWS spectrum as well as the availability and quality of the TVWS channels due to the presence of primary and secondary users. A new wireless communication architecture is proposed where the focal point is an aggregated robust wireless link (DSM Link) between the DSM engine and the DSM clients. Simulation results show significant gains in performance in terms of satisfied users, robustness and latency when compared with a conventional 802.11 system.
\end{abstract}

Index Terms - DSM, TVWS, Carrier Aggregation, 802.11

\section{INTRODUCTION}

As specified in the FCC rules [1], unlicensed devices operating within TVWS spectrum as secondary users are required to vacate their current channels upon the detection of a primary user. This requirement makes it difficult for a wireless communication operating in TVWS spectrum to guarantee service to its users. One way to help improve robustness when operating in TVWS spectrum is to design a communication system that can dynamically and frequently change the operating channel, with little or no interruption to the ongoing traffic. In an urban area, the available TVWS channels are often quite limited as there are many television broadcasters, and many wireless microphone users. However, within a local area such as a home or small office, the actual number of available TVWS channels may be more than that which is advertised in the FCC database. This could be as a result of the conservative algorithm used by the FCC database as well as due to localized fading within the small space. A communication system that incorporates sensing as defined by the FCC can take advantage of the increased availability of TVWS channels.

In this paper, we propose a wireless communication system that can operate reliably in TVWS spectrum. The proposed wireless communication system is composed of the following entities as shown in Fig. 1: A new central entity called the
DSM engine manages the interface to the TVWS database, and allocates spectrum dynamically among the devices or DSM clients operating in TVWS. The DSM clients are typical 802.11 devices but with additional functionality to operate reliably in TVWS spectrum. Both the DSM Engine and client have the functionality to sense and detect for primary users. The DSM system architecture is discussed in more detail in Section II. Section III explains the main functions and procedures mentioned above.

In the rest of the paper we describe how the DSM system sets up and maintains a robust and efficient DSM link to achieve the reliable system performance. Since the available TVWS channels are often sparse and discontiguous, the simultaneous use of discontiguous chunks of spectrum is critical to meet the required quality of service (QoS), and to maximize the bandwidth usable by a system or a user. A new MAC layer aggregation scheme based on 802.11 MAC/PHY protocols is proposed to achieve this. Section IV describes MAC layer aggregation over non-contiguous TVWS channels in detail. Section V presents simulation results showing performance improvement when compared with a non aggregated system. Section VI concludes this paper.

\section{DSM SYSTEM ARCHITECTURE}

In this section we introduce the architecture of the DSM system, which is shown in Fig. 1 with its major components described below.

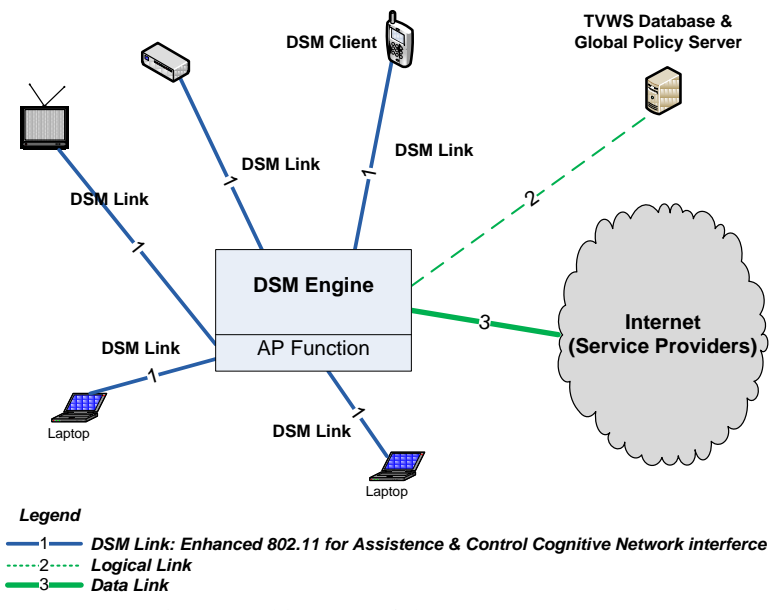

Fig. 1. Architecture of the DSM System 


\section{DSM Engine}

The DSM Engine manages all wireless communication between itself and the DSM clients occurring in the local area through the DSM link. The DSM Engine is interconnected to the external networks such as the cellular network, database and the IP networks through the WAN modem or wire-line link. The DSM Engine operates in the TVWS band as a mode II device as defined in FCC's Second Memorandum Opinion and $\operatorname{Order}^{[1]}$ (FCC 10-174), since it has access to the TVWS database and has geo-location capability. The DSM Engine can also operate in sensing only mode, which relies on spectrum sensing to determine a list of available channels (sensing only mode channels) in addition to those provided by the TVWS database. Operating in sensing only mode can potentially allow the DSM system to operate over a larger subset of channels than what the TVWS database allows.

\section{DSM Clients}

The DSM clients can operate as mode I devices as defined in [1], since they either do not have geo-location capability or access to the TVWS database. These devices will rely on the DSM Engine to indicate which channels can be used. Furthermore, a DSM client may also operate in a sensing only mode. In that case, for channels identified by the DSM Engine as sensing only mode channels, the DSM client will need to periodically verify that no primary user occupies these channels to enable transmission in these channels. The DSM Engine will schedule silent periods to enable adequate spectrum sensing on these channels at the DSM clients. Silent periods are coordinated time gaps created in the medium between the DSM engine and clients such that the medium is silenced to improve detection of primary users.

\section{DSM Link}

The communication link between the DSM engine and the DSM client is referred to as the DSM link and is used for synchronization of the DSM Engine and Clients, and for transfer of control and data plane traffic. We propose to implement the DSM link based on an enhanced 802.11 radio access technology (RAT) capable of operating over noncontiguous spectrum in TVWS.

\section{DSM SyStem FunCTIONAL ENTITIES AND PROCEDURES}

\section{A. DSM System Functional Entities}

In the proposed DSM system architecture, the DSM Engine is the new and core component which houses most of these functional entities. The DSM client has client versions of the functional entities. In the next few paragraphs, we explain the role of the logical functional entities in the DSM Engine and in the DSM clients, as illustrated in Fig. 2.

\section{Channel Management Function (CMF and CMF-C)}

The CMF is the central resource controller responsible for managing and allocating the radio resources efficiently to each of the devices and APs. It ensures that the DSM system operates reliably and efficiently under uncoordinated and heavy interference. This entity also manages the control channel, the admission control of clients and the centralized device database.

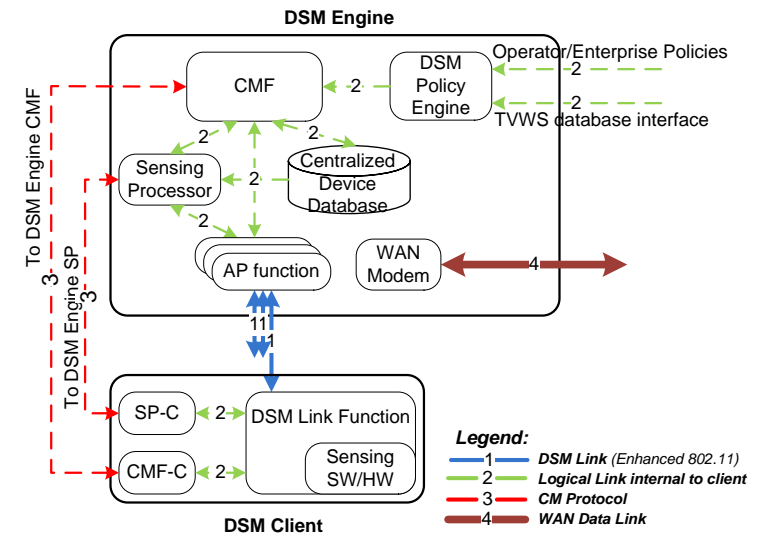

Fig. 2. The DSM Engine/Client Function Modules

The CMF-C is the main client entity that communicates directly with the CMF in the DSM Engine. It is responsible for obtaining channel resources from the CMF and ensuring that the resources are used and controlled according to the allocation rules determined by the CMF.

\section{Sensing Processor (SP and SP-C)}

The SP controls the sensing operation among the sensingcapable nodes in the network. It collects sensing information from the nodes and processes this information to facilitate the decision making in the CMF. In addition to using the SP to find and maintain the pool of available spectrum, the CMF instructs the SP to monitor specific bandwidth allocations that are actively being used by a device.

The SP-C is the main client entity that communicates directly with the SP in the DSM Engine. It controls the sensing operation and measurement reports configured by the SP on the client.

\section{AP Function}

The AP Function provides the main connectivity function for devices that join the network. It contains a coordination function which manages the spectrum aggregation based on the channels selected by the CMF.

\section{DSM Link Function}

The DSM Link Function provides the connectivity between the DSM clients and the AP function in the DSM Engine.

\section{Centralized Device Database (CDD)}

The Centralized Device Database stores device-specific information for all of the devices in the network that have been attached to the DSM Engine.

\section{DSM Policy Engine}

The DSM Policy Engine represents the enforcing entity of local regulatory rules and network operator rules within the DSM system.

\section{WAN Modem}

WAN Modem is integrated HW to provide connectivity between DSM system and the Internet or other WAN.

\section{B. DSM Procedures}

The following sub-section describes some of the key end to end procedures of the DSM system that are presented in the previous section. For each procedure, these descriptions 
provide a high-level view of the signaling between the logical functional entities.

\section{1) DSM Link Initialization}

Upon power-up of the DSM engine, the CMF will determine a pool of available channels and allocate from that pool a subset of aggregated channels to each of the AP functions. If the DSM Engine cannot allocate the number of channels it requires for the control channel based on the information in the TVWS database, it will use its sensing only capability to verify the usability of additional TV band channels for its control channel initialization.

In either case, the CMF will derive a list of initial channels to be used by the DSM system. The DSM Engine will then start sending control/management messages such as the beacon over the aggregated channels with the help of each AP. Special importance is given to the beacon, which is sent simultaneously over the aggregated channels with certain Information Elements (IEs) repeated over multiple channels and other Information Elements (IEs) segmented over the channels. The repetition of certain IEs allows a DSM client to discover and synchronize with the set of aggregated channels used by the DSM engine, by intercepting only a single channel. Note that the beacon message will also inform the DSM clients if one or more of the allocated channels are sensing only channels.

\section{2) Device Attachment and admission control}

Any device that joins the network must first attach with the $\mathrm{CMF}$ in order to make its presence known. The attachment process allows the DSM engine to control which devices are allowed into the DSM system based on continuous system performance monitoring by the CMF and available bandwidth/channels. It also allows the CMF to compile a list of clients under its direct management along with the location, capabilities, and properties of each client. A DSM Client initiating an attachment procedure must first associate with a discovered AP through a DSM link. In order to do this, a sensing stage may first be required in order to confirm the usability of any sensing only mode channels that are used by the DSM engine. Once association has been performed over a set of allowable channels the DSM client can send the attachment request. Upon confirming the success of the attachment procedure, the CMF will add the capabilities and supported service of the DSM client to the centralized device database. Once a client is registered in the device database, the SP can assign sensing tasks to that device in order to gain additional knowledge about current or future bandwidth utilization.

\section{3) Dynamic Channel Management}

The DSM system continuously maintains a list of candidate channels through the sensing function module and monitors the operational channel.

When a channel failure is detected by the AP function of the DSM Engine or by the DSM Link function of a client, a message is sent to the CMF notifying the failure. If one of the channels in the aggregation set encounters strong interference from other secondary users, CMF will do reassessment of the operational and candidate channels. In addition, the sensing processor notifies the CMF when a primary user is detected on one of the operational channels.

In each of these cases, the CMF confirms the need for a change of channel with the sensing processor and then allocates a new channel from the candidate channels list for use after checking with the policies from the policy engine. When the channel allocation has been made, a channel change message is sent by the CMF to the impacted AP function, which then relays this indication to the DSM clients over the aggregated channels.

The assumption in the procedure is that the failure does not happen on all channels simultaneously, and the control channel can be used to command the channel change. In the case of a widespread failure of all channels, the devices will need to re-associate on a different set of channels following a control channel initialization on a new set of channels.

The least interfered channel in the aggregation set is chosen as the primary channel in order to maximize system performance. The CMF continuously evaluates the channel quality of the aggregated channels set. When the non-primary channel quality is better than the primary channel quality with a certain hysteresis, a channel switch is triggered, the nonprimary channel becomes the primary channel and vice versa.

\section{DSM LINK - AGgREGATION}

With architecture, functions and procedures defined for the DSM system, this section addresses how the TVWS channels are used on the DSM link. In this section, we focus on the design principles for the DSM link based on an enhanced 802.11 RAT capable of operating over non-continuous TVWS spectrum in an aggregated fashion.

An example of MAC layer aggregation of up to four noncontiguous channels is shown in Fig. 3. There are four PHY chains, each handling the baseband signal processing of a carrier. The wideband digital transceiver simultaneously and independently modulates/demodulates corresponding signals. For transmission, the data stream is segmented into the number of active PHY chains and at the destination node, the data stream will be aggregated from the four independent PHY chains.

MAC layer aggregation requires the device to be capable of operating on these channels simultaneously without interfering with each other. However, the DSM clients cannot have simultaneous transmission on one aggregate channel and reception on other channels in the aggregation set due to self jamming of the wideband transceiver. This makes it necessary to synchronize the channel status over all the aggregated channels, i.e. either all the aggregated channels are on transmission/idle mode or all on receiving/idle mode.

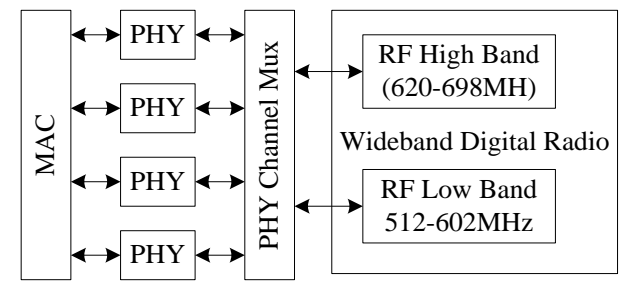

Fig. 3. MAC Layer Aggregation 


\section{1) Wideband Digital Radio}

The TVWS spectrum has a bandwidth of approximately $200 \mathrm{MHz}$, ranging from $512 \mathrm{MHz}$ to $698 \mathrm{MHz}$. The current state of the digital transceiver technology employs two RF chains to cover TVWS band, with the low band board covering $512 \mathrm{MHz}$ to $608 \mathrm{MHz}$ and the high band board covering $614 \mathrm{MHz}$ to $698 \mathrm{MHz}$ as shown in Fig. 3. Each board can aggregate up to 4 contiguous or non-contiguous spectrum segments with each $5 \mathrm{MHz}$ wide. The radios can also be configured to operate in sensing mode which allows measurement to be taken for detection of primary users.

\section{2) Channel Access Mechanism - Primary CSMA}

With the above mentioned limitation about simultaneous transmission and reception of aggregated channels, plus a basic requirement that all the devices get a fair chance of accessing the medium and that the chances of collision should be minimized, we use a channel access mechanism called Primary CSMA. The concept is to choose one of the channels from the aggregation set as the primary channel to perform CSMA with the assumption that all the other channels have the same channel status (busy or idle) as the primary channel. The choice of primary and non-primary channels is broadcast to the DSM clients through the beacon control message. As a result, once the client or the AP in DSM engine receives access to the primary channel, it also receives access to all of the channels in the set. The device/DSM engine on gaining access to the primary channel also contends for the nonprimary channels (secondary, tertiary and quaternary channels) for a period of PIFS before transmission to ensure that all four channels are in fact free. This behavior is illustrated in Fig. 4. The concept of the primary channel has been leveraged from the 802.11ac standard.

In the case that contention fails for one of the non-primary channels the transmission will still occur over the remaining channels. It is only when the primary channel is busy that the entire transmission is deferred to a subsequent transmission opportunity. This procedure is called defer/5/10/15/20, as it allows the option of transmitting over one, two, three or all four of the TVWS channels depending on availability.

For data transmission and retransmission over the aggregated channels, two rules are applied:

Rule \#1: The DSM link in the system is used for both control plane messages and user plane traffic, i.e. the aggregated channels are shared over time and both control and data have to contend for the access to the channels.

Rule \#2: The DSM client or AP function in DSM engine gains access to all of the four aggregated channels through the primary channel CSMA mechanism if it has control messages or user traffic to send. The destination of the transmissions could be different, but for simplicity the destination can be limited to one.

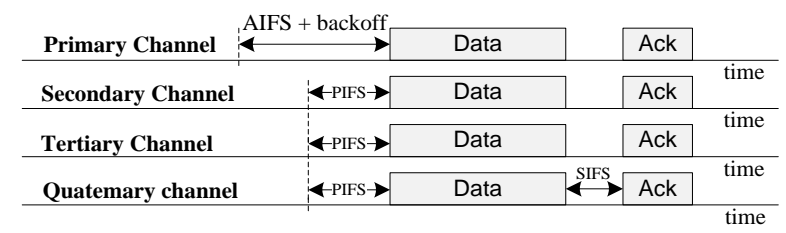

Fig. 4. Primary Channel CSMA mechanism

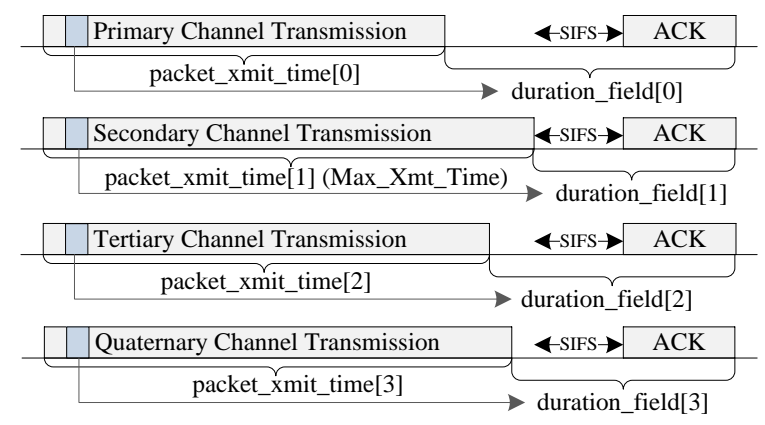

duration_field[i] $=($ Max_Xmt_Time-packet_xmit_time[i] $)+($ SIFS + ACK $)$ time

Fig. 5. Example of Common Virtual Carrier Sensing

\section{3) Acknowledgement Synchronization}

Since a device cannot transmit and receive at the same time, even on different channels, this makes it necessary to synchronize the acknowledgement procedures performed by the receiver over the aggregated channels. For example, a device could not receive an acknowledgement on a given channel while still being busy transmitting over another channel. However, care must be taken when synchronizing the ACKs as it may result in idle times between the data transmission and ACK transmission, during which other devices could attempt to access the channel. A new method called Common Virtual Carrier Sensing is proposed to solve the synchronization issue.

In 802.11 , the medium is determined to be idle only when both the physical and virtual carrier sense mechanisms indicate it to be so. The virtual carrier sense mechanism is used to check the medium busy duration information set in MAC header or RTS/CTS frames.

In the aggregated channel case, at the transmission side, if the medium busy duration information of each channel is changed to represent the busy status of the channel that has longest transmission end time, instead of its own channel busy status, then unexpected channel access on the idle mediums during ACK synchronization process can be avoided. This ensures that this new virtual carrier sensing mechanism (Common Virtual Carrier Sensing) on all the channels return the same result. An example of Common Virtual Carrier Sensing is shown in Fig. 5.

\section{DSM SYSTEM - PERFORMANCE}

\section{1) Test Bench Description}

The goal of the test bench is to: study the performance of MAC layer aggregation against a comparable system that does not use aggregation, and verify the performance of the channel access and ACK synchronization mechanism used to support aggregation.

The test bench is based on OPNET simulation software. It is composed of one DSM engine (manages 4 TVWS channels), TVWS database, gateway, interference source, server and several DSM clients supporting various traffic applications (Email, FTP, HTTP, voice and video) in an area of $10 \mathrm{~m} \times 10 \mathrm{~m}$ as shown in Fig. 6. MAC layer aggregation is implemented based on the $802.11 \mathrm{~g}$ MAC specification. A data rate of $12 \mathrm{Mbps}$ was used as the nominal data rate for the $5 \mathrm{MHz}$ bandwidth channel corresponding to approximately $1 / 4$ the maximum data rate of 
$20 \mathrm{MHz}$ bandwidth channel. Free space path loss model is used and transmission powers of the AP and the DSM clients were set strong enough for them to communicate with each other without any errors. We also assumed that four non-contiguous TVWS channels were always available for aggregation. The interference source is introduced to generate interference in some simulation scenarios that evaluate interference impact.

We used a similar system without channel aggregation as our comparison baseline. In the baseline scenario, the DSM engine is replaced by four independent APs. We assume the DSM clients are evenly distributed amongst the four APs. The DSM link is replaced by four independent links each utilizing one TVWS channel.

\section{2) Performance Results}

Various traffic models are used to examine the DSM system performance from aspect of overall system throughput, user application latency and number of satisfied clients.

To simplify the analysis of the simulation results, scenarios with traffic load composed of UDP applications are run separately from those composed of TCP applications.

\section{a) Application Latency}

The UDP Application latency is defined as frame end to end delay which is calculated from the time one frame is generated to the time when it is successfully received at the receiver end.

The UDP application traffic model is listed in Table I. Each voice frame size is small so that no fragmentation will be done and each frame occupies one channel for transmission. In contrast each video frame size is large such that it will be fragmented to more than four MPDUs because of the maximum MPDU size limitation. In the channel aggregation case, four MPDUs will be transmitted simultaneously while in the non-aggregation channel case only one MPDU can be transmitted in every transmission slot.

The results are shown in Table III. Voice application latency is similar in both the aggregation and non-aggregation case. Video application latency in the aggregation case is much shorter compared with the non-aggregation case.

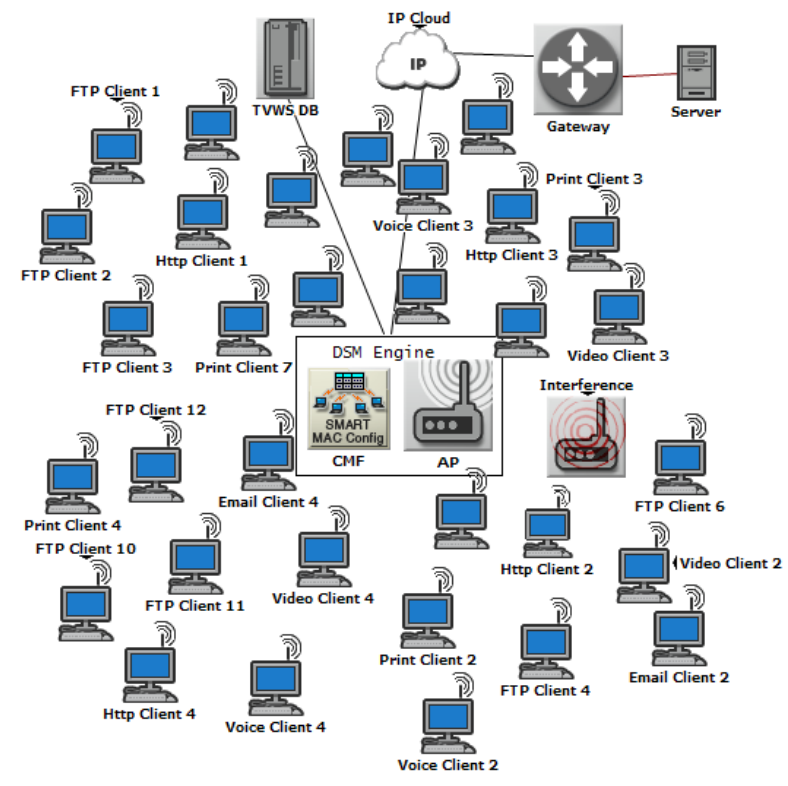

Fig. 6. OPNET DSM System Test Bench
Table I UDP APPLICATION TRAFFIC MODEL

\begin{tabular}{c|c|c}
\hline Applications & Num of Clients & Traffic load per Client \\
\hline Voice & 4 & 30kbps bi-directional \\
\hline Video Conference & 4 & $1.39 \mathrm{Mbps}$ bi-directional \\
\hline
\end{tabular}

\begin{tabular}{|c|c|c|c|}
\hline Applications & $\begin{array}{l}\text { Num of } \\
\text { Clients }\end{array}$ & Traffic Description & $\begin{array}{c}\text { Application interval time } \\
\text { distribution }\end{array}$ \\
\hline FTP & 12 & $\begin{array}{l}\text { file size } 3 \text { Mbytes, } 75 \% \\
\text { download, } 25 \% \text { upload }\end{array}$ & $\begin{array}{c}\text { Poisson distribution with } \\
\text { a mean of } 15 \mathrm{sec}\end{array}$ \\
\hline Email & 4 & $\begin{array}{c}\text { email size } 2 \text { Kbytes, } \\
\text { average } 3 \text { emails in the } \\
\text { queue when send or receive }\end{array}$ & $\begin{array}{l}\text { Exponential distribution } \\
\text { with a mean of } 60 \mathrm{sec}\end{array}$ \\
\hline $\begin{array}{c}\text { Web } \\
\text { Browsing }\end{array}$ & 8 & $\begin{array}{l}1 \text { Kbytes page contents and } \\
5 \text { medium size images }\end{array}$ & $\begin{array}{l}\text { Exponential distribution } \\
\text { with a mean of } 30 \mathrm{sec}\end{array}$ \\
\hline Print & 4 & $\begin{array}{c}\text { file size: normal } \\
\text { distribution with a mean of } \\
30 \mathrm{k} \text { bytes and variance } 9 \mathrm{M} \text {. }\end{array}$ & $\begin{array}{l}\text { Exponential distribution } \\
\text { with a mean of } 180 \mathrm{sec}\end{array}$ \\
\hline
\end{tabular}

\begin{tabular}{l|c|c}
\multicolumn{3}{c}{ Table III APPLICATION LATENCY } \\
\hline DSM Link & with Aggregation & without Aggregation \\
\hline Voice Frame end to end delay & $83.777 \mathrm{~ms}$ & $83.074 \mathrm{~ms}$ \\
\hline Video Frame end to end delay & $12.638 \mathrm{~ms}$ & $30.779 \mathrm{~ms}$ \\
\hline FTP upload time & $4.946 \mathrm{~s}$ & $7.526 \mathrm{~s}$ \\
\hline FTP download Time & $4.778 \mathrm{~s}$ & $7.526 \mathrm{~s}$ \\
\hline Email upload Time & $0.089 \mathrm{~s}$ & $0.192 \mathrm{~s}$ \\
\hline Email download time & $0.104 \mathrm{~s}$ & $0.193 \mathrm{~s}$ \\
\hline HTTP page response time & $0.071 \mathrm{~s}$ & $0.160 \mathrm{~s}$ \\
\hline
\end{tabular}

The TCP applications traffic model is listed in Table II. The TCP application either has large sized data that needs to be fragmented to many MPDUs or has many queued small sized data. In the aggregation case, they can be transmitted simultaneously on aggregated channels so that the application response time is much faster than the non-aggregation case, as shown in Table III.

\section{b) Satisfied Clients}

In this subsection we describe more simulation scenarios and results that evaluate the performance of MAC layer aggregation with respect to satisfied clients.

A video client is considered satisfied if the average video frame end to end delay is less than 150ms. A FTP client is considered satisfied if the file transfer is completed with 10 seconds. The video and FTP client traffic profile are listed in Table IV.

Table IV Traffic Profile in Satisfied Client Scenario

\begin{tabular}{c|c|c}
\hline Applications & $\begin{array}{c}\text { Per call/transfer traffic } \\
\text { description }\end{array}$ & $\begin{array}{c}\text { Time interval between } \\
\text { calls/transfers }\end{array}$ \\
\hline FTP & $\begin{array}{c}\text { File size: exponential distribution } \\
\text { with a mean of 5M bytes. } \\
75 \% \text { of the time doing download } \\
\text { and 25\% of the time doing upload }\end{array}$ & $\begin{array}{c}\text { Poisson distribution } \\
\text { with a mean of } 60 \mathrm{sec}\end{array}$ \\
\hline $\begin{array}{c}\text { Video } \\
\text { Conference }\end{array}$ & $\begin{array}{c}1.39 \mathrm{Mbps} \text { bidirectional. } \\
\text { call duration: exponential } \\
\text { distribution with a mean of } 45 \mathrm{sec}\end{array}$ & $\begin{array}{c}\text { Exponential } \\
\text { distribution with a } \\
\text { mean of } 180 \mathrm{sec}\end{array}$ \\
\hline
\end{tabular}



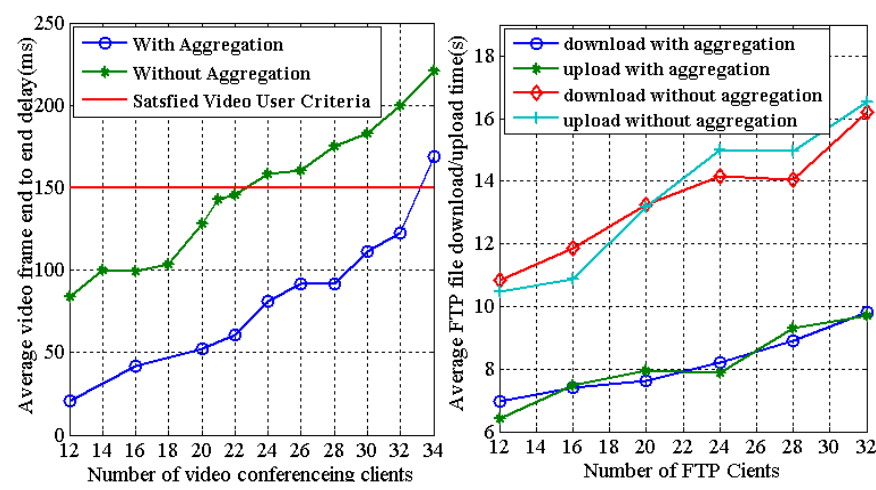

Fig. 7. Number of Satisfied Video/FTP Clients

The simulation results are shown in Fig. 7. It shows that in order to meet the satisfied client criteria, for video applications, the capacity of the DSM system must be limited to 32 video clients in the aggregation channel case and 22 video clients in the non-aggregation channel case; for FTP applications, the capacity of the DSM system is limited to 32 FTP clients in the aggregation channel case and less than 12 FTP clients in the non-aggregation channel case.

\section{c) Interference Impact}

In this simulation subsection, we will evaluate the system performance under interference. We assume that the DSM system is under high traffic load, one of the four aggregated channels experiences a constant interference. The interference level of one channel varies in different simulation runs. We use $0 \%, 20 \%$ and $40 \%$ interference levels which correspond to the percentage of time the channel experiences interference. The interference occurs approximately every $50 \mathrm{~ms}$. Furthermore we assume that no candidate TVWS channel is available to replace the channel experiencing interference.

Simulation results are listed in Table VI. With 20\% interference level the performance (application latency) degradation is not obvious in both aggregation channel or nonaggregation channel case. While when interference level is increased to $40 \%$, application latency is significantly degraded in the non-aggregation channel case, and has some small impact in the aggregation case.

Table V Traffic Profile in Interference Scenario

\begin{tabular}{c|c|c}
\hline Applications & $\begin{array}{c}\text { Num of } \\
\text { Clients }\end{array}$ & Traffic load per Client \\
\hline FTP & 12 & $\begin{array}{c}\text { file size 3Mbytes, 75\% download, 25\% } \\
\text { upload, interval time between 2 transfers: } \\
\text { Poisson distribution with a mean of 13sec }\end{array}$ \\
\hline Video Conference & 8 & 1.39Mbps bidirectional \\
\hline
\end{tabular}

Table VI InTERFERENCE IMPACt ON APPLICATION LATENCY

\begin{tabular}{c|c|c|c|c|c|c}
\hline \multirow{2}{*}{$\begin{array}{c}\text { Interference } \\
\text { Level }\end{array}$} & \multicolumn{3}{|c|}{ With Aggregation } & \multicolumn{3}{c}{ Without Aggregation } \\
\cline { 2 - 7 } & Video & $F T P$ DL & $F T P$ UL & Video & FTP DL & $F T P U L$ \\
\hline $0 \%$ & $26.3 \mathrm{~ms}$ & $5.51 \mathrm{~s}$ & $5.42 \mathrm{~s}$ & $35.8 \mathrm{~ms}$ & $7.36 \mathrm{~s}$ & $7.40 \mathrm{~s}$ \\
\hline $20 \%$ & $28.3 \mathrm{~ms}$ & $5.69 \mathrm{~s}$ & $5.70 \mathrm{~s}$ & $40.5 \mathrm{~ms}$ & $7.80 \mathrm{~s}$ & $8.45 \mathrm{~s}$ \\
\hline $40 \%$ & $32.4 \mathrm{~ms}$ & $6.50 \mathrm{~s}$ & $7.00 \mathrm{~s}$ & $278.0 \mathrm{~ms}$ & $22.3 \mathrm{~s}$ & $32.1 \mathrm{~s}$ \\
\hline
\end{tabular}

d) Dynamic Channel Switch

The previous simulation results show that the DSM system is quite robust to interference when compared to the baseline system, but there is still some performance degradation when the interference level increases. In this subsection, the dynamic channel switch feature is enabled to show the performance improvement in an interference environment.

We assume that the interference is present on all the operational and candidate channels. The sensing device monitors both the operational channels and candidate channels for their interference levels and there are always available candidate channels. Two dynamic switch algorithms are applied. One is Primary Channel Switch which replaces the primary channel with the least interfered channel in the aggregation set. The other one is Channel Switch which replaces an operational channel with a better channel from the candidate set, when the interference level experienced in one of the operational channels crosses a threshold i.e. in this simulation the threshold is $25 \%$.

The results are shown in Fig. 8. With "Interference without Channel Switch" scenario as lower bound and "No Interference" scenario as upper bound, we see throughput increases a little bit with primary channel switch enabled, increases significantly with channel switch enabled and almost reaches the upper bound when both switch algorithms are turned on.

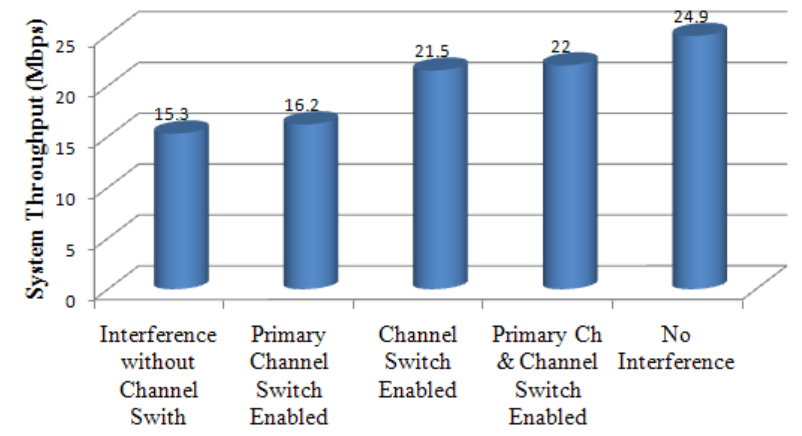

Fig. 8. Throughput Improvement with Dynamic Channel Switch Enabled

\section{CONCLUSION}

We developed a wireless communication system that uses DSM to opportunistically and efficiently operate in TVWS spectrum and can be deployed in a home or small office environment. This paper describes architecture and spectrum aggregation technique that achieves significant performance gains compared to independent use of multiple TVWS channels. In particular, we enhanced the 802.11 MAC/PHY protocol to support MAC level aggregation of multiple non contiguous channels in TVWS to increase both performance and robustness. The simulation results show that our proposed aggregated DSM link achieves significant performance gains in terms of system throughput, application latency, and user satisfaction. We also showed that further gains are achievable by dynamically switching the TVWS channels with respect to interference.

\section{REFERENCES}

[1] FCC Second Memorandum Opinion and Order (FCC 10-174)

[2] IEEE Std 802.11-2007, Wireless LAN Medium Access Control (MAC) and physical Layer (PHY) Specifications 